\title{
Primarysplenic Large Cell B Lymphoma: Case Report
}

\author{
Asmaa Lakhdissi*, Khadija Bellahammou, Narimane Salmi, Hamza Ettahri, Mustapha El Kabous, \\ MeryemAit lhaj, Sihame Lkhoyaali, Hind Mrabti, Hassan Errihani
}

Department of Medical Oncology, National Institute of Oncology, Rabat, Morocco

*Corresponding Author: Asmaa Lakhdissi, Department of Medical Oncology, National Institute of Oncology, Rabat, Morocco, E-mail: asmaalakhdissi@gmail.com

\begin{abstract}
Background: Primary splenic lymphoma is a rare disease with heterogeneous definition, compromising less than $1 \%$ of non-Hodgkin's lymphoma.

Case presentation: We are reporting a case of 50 year old man who presented splenomegaly, fever, weight loss and night sweats. The investigations revealed a splenomegaly with splenic lesion which cancorrespond to abscess. The patient underwent splenectomy for definitive diagnosis and treatment. Diagnosis of primary diffuse large B cell lymphoma of the spleen was made and confirmed by immunochemistry and the patient received 6 cycles of RCHOP (Rituximab + cyclophosphamide + Adriamycin + vincristine + prednisone) and he is currently well and in complete remission more than 1 year after diagnosis.

Conclusion: Primary splenic lymphoma is rare; splenectomy is an important step in its diagnosis and treatment. Early splenectomy combined with chemotherapy is an optimal strategy.
\end{abstract}

Abbreviations: PSL: Primary Splenic Lymphoma; RCHOP: Rituximab/Cyclophosphamide/Adriamycin/Vincristine/Prednisone; ESR: Erythrocyte Sedimentation Rate; CRP: C Reactive Protein; LDH: Lactate Dehydrogenase; DLBC: Diffuse Large B Cell Lymphoma; ALCL: T Cell Large Anaplastic Lymphoma; MRI: Magnetic Resonance Imaging
Received Date: January 31, 2016

Accepted Date: April 04, 2016

Published Date: April 08, 2016

Citation: Lakhdissi, A,. et. al. Primarysplenic Large Cell B Lymphoma: Case Report. (2016) J Gastro Dis Liver Func 2(2): 73- 76

DOI: $10.15436 / 2471-0601.16 .765$

Keywords: Primary splenic lymphoma; Splenectomy; Chemotherapy

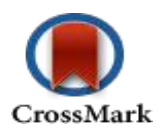

\section{Background}

The spleen is a lymphoid organ that is frequently invaded by malignant lymphomas ${ }^{[1]}$. However, primary splenic lymphoma is unfrequently and very rare, the incidence is about $1 \%{ }^{[2]}$, it has been misdiagnosed as splenic abscess in the past ${ }^{[3]}$ and the initial diagnosis is often taking place after splenectomy. The definition in the literature still heterogeneous, if we take the criteria proposed by Das Gupta et al, the diagnosis of primary splenic lymphoma should be made when the disease is confined to spleen or at the most involves hilar lymph nodes with no recurrence of disease after splenectomy ${ }^{[4]}$.

This case report highlights a case of primary splenic diffuse large B cell lymphoma revealed by splenomegaly and spleen lesion mimicking an abscess and illustrates the management of the case.

\section{Case report}

A 50 year old man, without pathological antecedents, was admitted in February 2014 to the hospital with three month history of abdominal pain, weight loss, fever and night sweats. The clinical examination found splenomegaly. There was no hepatomegaly, no ascites and no palpable lymphadenopathy.

Laboratory findings showed hemoglobin level of $11.1 \mathrm{~g} / \mathrm{dL}$, leukocyte count of $10.71 \mathrm{x} 10^{3} / \mathrm{ml}$ and platelet count of 250000 /ml.ESR, CRP, LDH, renal and markers of hepatic function were within normal limits. 
A computed tomography of abdomen and chest showed a splenomegaly with one lesion which can correspond to abscess without evidence of other lesions or thoracic or abdominal adenopathy (Figure 1).

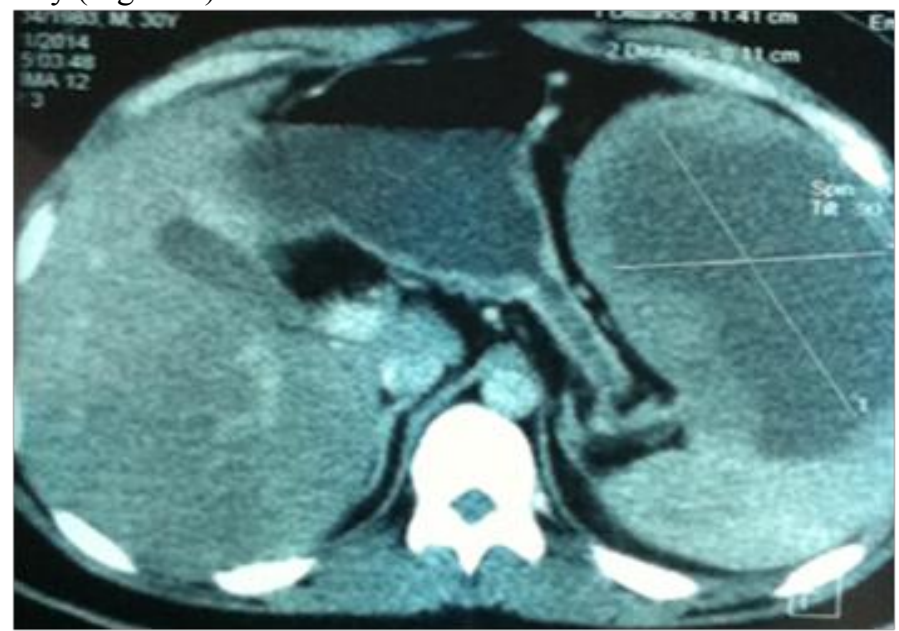

Figure 1: Computed tomography showing massive splenomegaly with splenic lesion may correspond to abscess.

Afine needle biopsy was not performed because our patient suffers from worsening abdominal discomfort and pain due tosplenomegaly. An emergencyopen splenectomy was planned and there was not a postoperativecomplication.

A diagnosis of primary splenic lymphoma was made after splenectomy. The resected spleen weighed 731 gram and it measures 14 x $9 \mathrm{~cm}$ with one lymph node in splenic hilum. The examination of the spleen findsthat the tumor was completely confined to splenic capsule without extra splenic extensionand there was no purulent or other type liquid content lesion.

Microscopy revealed malignant tumor formation characterized by a diffuse proliferation of monotonous population of large neoplastic lymphoid cells (large B cell lymphoma)

(figure 2).

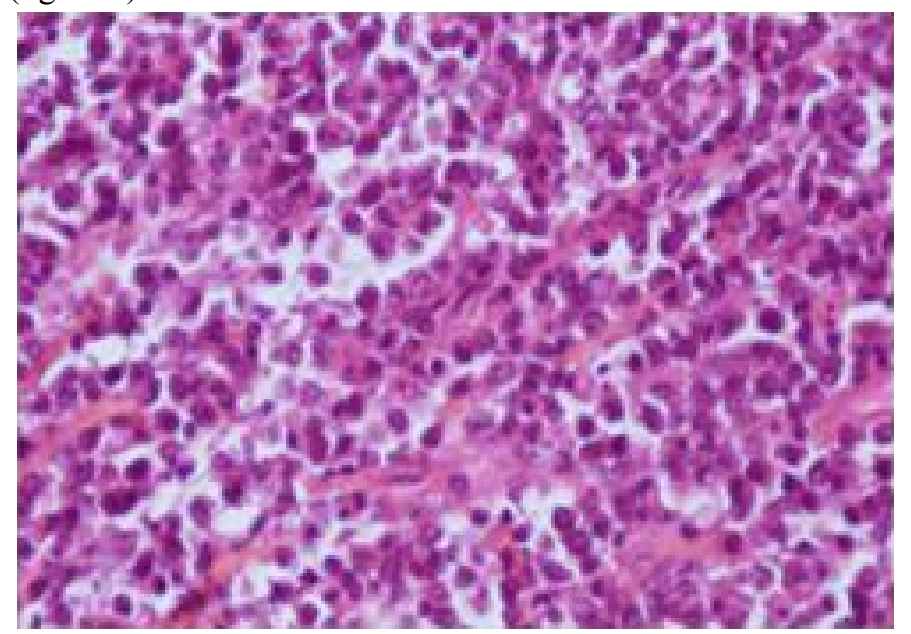

Figure 2: Diffuse large B cell type

The tumor cells were immunopositive for CD20, CD79a that confirmed the diagnosis of large B cell lymphoma (figure 3 and 5) and negative for CD10, Cyclin D1 Bcl2 and KI67 > 70\%(figure 4)

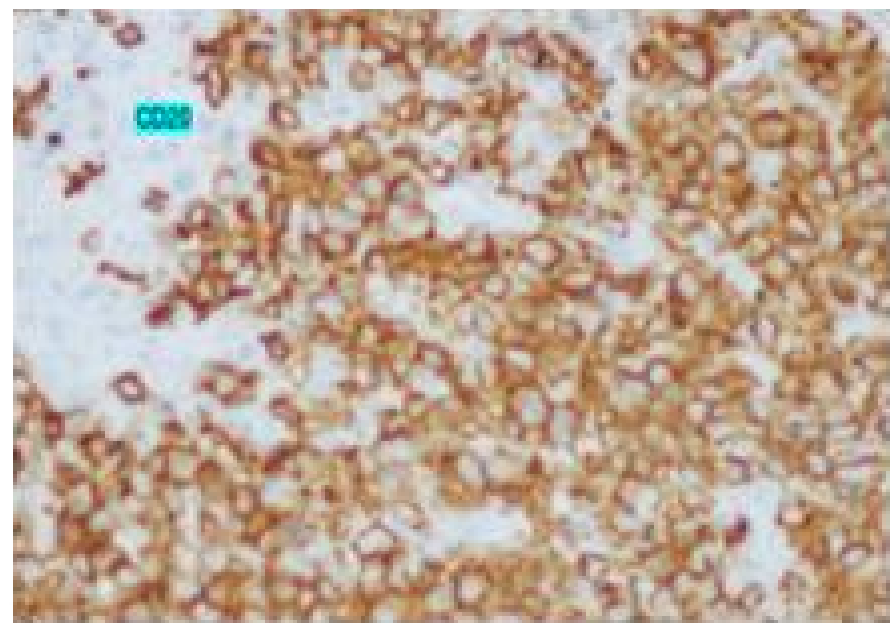

Figure 3: CD20+ positive lymphoid cells

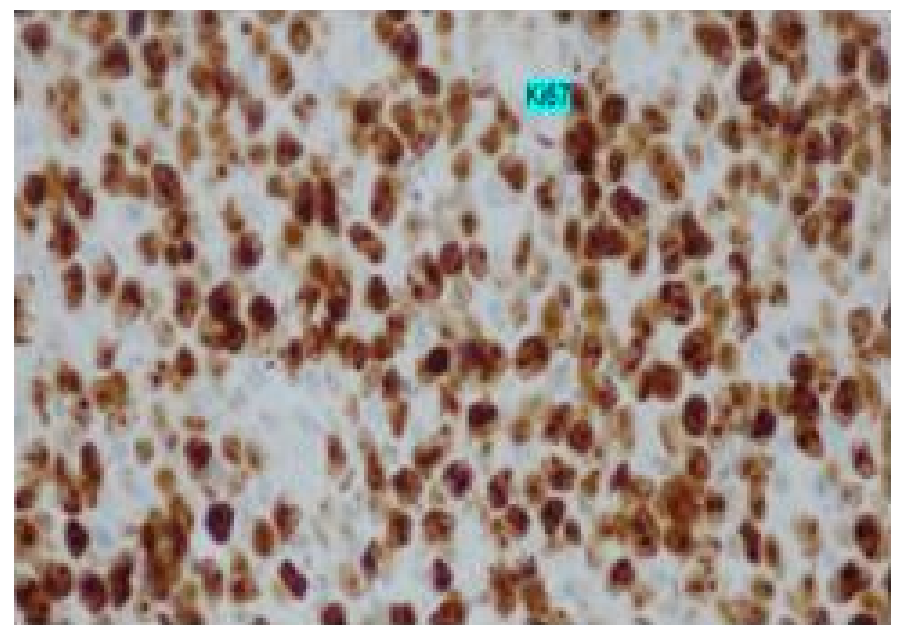

Figure 4: proliferation Ki67

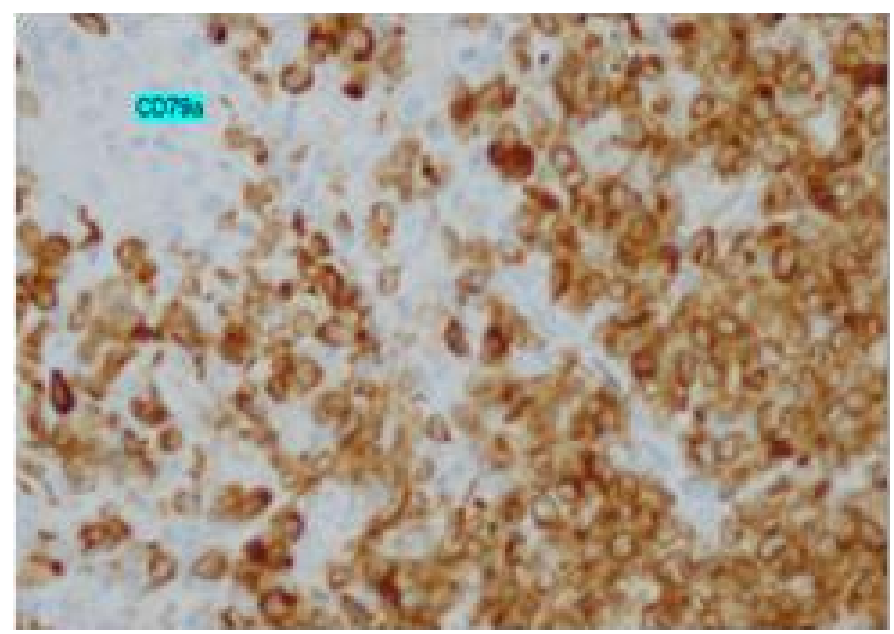

Figure 5: CD79a positive lymphoid cells

The bone marrow biopsy was normal. According to The Ann Arborstaging, the patient was staged IsBa.

Six courses of chemotherapywith CHOP (Adriamycin + Cyclophosphamide + Vincristine + prednisone) plus rituximab was given, the cycle was repeated after 21 days and Completed remission has continued more than 1 year post-operative without complication related to disease and splenectomy. 


\section{Discussion}

The spleen is the organ that is responsible for purifying the blood as well as storing blood cells and serves a valuable role in immune function.

More than half of patients affected by Hodgkin disease and about third with non-Hodgkin lymphoma have splenic involvement ${ }^{[5]}$.

Primary lymphoma of the spleen (PSL) is very rare, the incidence is less than $1 \%$ and the definition in the literature is ambiguous and vaster way. Das Gupta et al first defined this condition as confined to the spleen or hilar lymph nodes with no recurrence of disease for at least 6 months after splenectomy ${ }^{[4]}$ .On the Other hand, Skarin et al defined PSL as lymphoma with splenic involvement in which splenomegaly is the dominant feature $^{[6]}$. For Dachmannet al, PSL is defined by hypodense splenic lesions on contrast enhanced CT scans or hypoechoic lesions on ultrasound on imaging studies ${ }^{[7]}$. Whereas, for Ahmann and Kiely, stageI refers to disease confined to the spleen,stageII implies involvement of the spleen and hilar lymph nodes and stageIII refers to extra-splenic nodal or hepatic involvement ${ }^{[2]}$.

Consequently, literature review doesn't allow determining the actual frequency of primary splenic lymphoma. This is mainly due to the lack of a standard definition when performing diagnosis.

The most common clinical presentation of PSL is left upper quadrant pain, waist pain, weight loss and fever. However, these symptoms are no-specific.Cytopenia can also be a presenting feature.

The most common appearance of PSL on diagnosis imaging studies is hypodense lesions on contrast-enhanced CT scans and hypoechoic lesions on sonography ${ }^{[8]}$. MRI is also a useful tool for identifying and characterizing focal splenic lymphoma $^{[9]}$.

Interestingly, patients with PSL mentioned in literature showed splenomegaly without exception and splenectomy was the method of diagnosis and treatment ${ }^{[10,11]}$.

On the other hand, splenic needle biopsy may provide an adequate diagnosis for PSL without severe complications ${ }^{[12]}$ and can help to make differential diagnosis of a solitary splenic mass such as hemangioma, hamartoma and abscess, as well as metastatic disease ${ }^{[13]}$.

Majority of splenic lymphoma are of B-cell origin, with the most common histologic diagnosis being a low-grade or intermediate-grade lymphoma ${ }^{[5]}$. Other subtypes are diffuse large B cell lymphoma (DLBC) and T cell large anaplastic lymphoma (ALCL). Our patients was a case of diffuse large B cell lymphoma

Treatment of PSL still debate due to the lack of clinical trials. Possible treatment modalities include splenectomy, systemic chemotherapyand local radiotherapy. Radiotherapy is an option for patients diagnosed with PSL if splenectomy isn't feasible.

Morel et aldiscussed the role of early splenectomy in malignant lymphomas with prominent splenic involvement (primary lymphomas of the spleen). The outcomes were analyzed retrospectively of 59 cases of non-Hodgkin lymphoma. Forty of the 59 patients underwent splenectomy. Twenty-nine splenectomized and 16 patients whose spleens were not removed received chemotherapy or radiation therapy. The median actuarial surviv- al was 108 months in splenectomized patients and 24 months in those not treated surgically $(\mathrm{P}=0.0001)$. Thus;splenectomy appears to play a role in the treatment of primary spleen lympho$\mathrm{ma}^{[14]}$.

A study of 104 patients with primary low grade non-Hodgkin lymphoma of the spleen concluded that early splenectomy along with the combination of chemotherapy was the optimal treatment due to higher rates of remission, a more prolonged duration of remission and better overall survival rates when compared with splenectomy or splenectomy with a single agent chemotherapy ${ }^{[15]}$.

Most cases of aggressive subtype (DLBC, ALCL) show disease expansion and progression, requiring appropriate chemotherapy.

The CHOP regimen appears to be the most widely accepted chemotherapy regimen for PSL ${ }^{[16]}$, despite the lack of clinical trials of the subject.

Our patient was treated after splenectomy with 6 cycles of Rituximab (anti CD20) +CHOP with favorable evolution and still complete remission after more than one year of diagnosis.

\section{Conclusion}

For the case of patients showing fever or any other symptoms that are related to radiographic signs of single or multiple focal lesions in the spleen, diffuse large cell lymphoma is to be considered when performing differential diagnosis.Splenectomy is the most popular choice because it provides both correct diagnosis and effective treatment Splenectomy combined to chemotherapy is the optimal strategy for the aggressive subtypes.

Consent: An informed consent was obtained from the patient to write this case report.

Competing Interests: The authors declare that they have no competing interests.

Authors' contribution: All authors contributed to the literature research and drafting of the manuscript.

All authors read and approved the final manuscript.

Acknowledgment: We thank our colleagues from national institute of oncology Rabat Morocco who provided insight that greatly assisted the research.

\section{References}

1. Thomas, J.L., Bernardino, M.E., Vermess, M, et al. EOE 13 in the detection of hepato-splenic lymphoma. (1983) Radiology 145(3): 629-634.

2. Ahmann, D.L., Kiely, J.M., Harrison, E.G., et al. (1966) Malignant lymphoma of the spleen. A review of 49 cases in which the diagnosis was made at splenectomy. Cancer 19(4): 461-469. 3. Huggett, M.T., Howieson, A., Wakefield, C., et al. An apparent splenic abscess. (2005) J R Soc Med 98(10): 474- 475.

4. Das Gupta, T., Goombes, B., Brosfeld, R.D. Primary malignant neoplasms of the spleen. (1969) Surg Gynecol Obstet 120: 947-960.

5. Gobbi, P.G., Grignani, G.E., Pozzetti, U., et al. Primary splenic lymphoma:Does it exist? (1994) Haematologica 79(3): 286-293. 
6. Skarin, A.T., Davey, F.R., Maloney, W.C. Lymphosarcoma of the spleen.Results of diagnostic splenectomy in 11 patients. (1971) Arch Intern Med 127(2): 259-262.

7. Dachman, A.H., Buck, J.L., Krishnan, J., et al. Primary non-Hodgkin's splenic lymphoma. (1998) Clin Radiol 53(2): 137-142.

8. Lee, J.D., Park, C.H., Griffith, J., et al. Gallium-67 scintiscan in the diagnosis of primary splenic non-Hodgkin's lymphoma after the treatment of Hodgkin's disease. (1992) J Nucl Med 33(6): 1183-1185.

9. Ioannidis, O., Papaemmanouil, S., Paraskevas, G., et al. Pathologic Rupture of the Spleen as the Presenting Symptom of PrimarySplenic Non-Hodgkin Lymphoma. (2011) J Gastrointest Cancer 43(1): S9-S12.

10. Brox, A., Bishinsky, J.I., Berry, G. Primary non-Hodgkin lymphoma of the spleen. (1991) Am J Hematol 38(2): 95-100.

11. Falk, S., Stutte, H.J. Primary malignant lymphomas of the spleen.A morphologic and immunohistochemical analysis of 17 cases. (1990) Cancer 66(12): 2612-2619.
12. Hsu-Heng, Y., Yang-Yaun, C., Maw-Soan, S. Ultrasound-guided Fine Needle Biopsy of Splenic Lymphoma. (2003) J Med Ultrasound 11: 71-75.

13. Choi, P., Park, S.W., Kang, M.J., et al. A case of primary splenic lymphoma presenting as a splenic abscess. (2009) Korean J Med 76(1): S49-S53.

14. Morel, P., Dupriez, B., Gosselin, B., et al. Role of early splenectomy in malignant lymphomas with prominent splenic involvement (primary lymphomas of the spleen). A study of 59 cases. (1993) Cancer 71(1): 207-221.

15. Musteata, V.G., Corcimaru, I.T., Iacovleva, I.A., et al. Treatment options for low-grade non-Hodgkin's lymphomas. (2004) Clin Lab Haematol 26(6): 397-401.

16. Healy, N.A., Conneely, J.B., Mahon, S., et al. Primary splenic lymphoma presenting with ascites. (2011) Rare Tumors 3(2): e25. 